\title{
Simultaneous Sampling and HPLC Injection from USP Apparatus 4 using a Dual Sampling Rack
}

\author{
Dr. Andrew Hodson ${ }^{1}$ and Keith A Wilkinson ${ }^{1,2}$
}

T he advantages of the USP Apparatus 3 and 4 are that they lend themselves to extended controlled release products and are particularly suited for change of media; hence control of $\mathrm{pH}$ throughout the test. The USP Apparatus 4 in particular can be used for low solubility products since by design there is no limit to the volume of media that can be used for each sample.

Analysis using these apparatus can be long, up to and above 24 Hours in many cases.

USP Apparatus 4 requires the sampling pump to be on continuously throughout the analysis, as the dissolution rate is directly proportional to the flow rate of media that is pumped into the flow through cell. Sampling for this technique therefore requires that continuous collection or measurement of the eluted sample be maintained.

Conventional sampling techniques can be one of two methods, dependent upon whether the sample can be detected using direct UV measurement in a spectrophotometer or requires HPLC analysis.

For direct UV measurement, samples can be pumped through a spectrophotometer and the Absorbance data integrated with time to calculate the amount of substance dissolved. The frequency of sampling will depend on the dissolution characteristic of the sample and can be as low as a 1-minute interval. If, however, the concentration of the sample is too high to be measured directly using a Spectrophotometer, then the samples must be collected first then measured manually after the dissolution run has completed.

Until recently, for HPLC detection, the only option was to collect samples in a fraction collector. Difficulties can arise due to the large volume of eluent to be collected. With typical flow rates of $16 \mathrm{ml} / \mathrm{min}$, large tube sizes are required. As the dissolution time increases, larger sample storage may be required which may not be practical. Fraction collectors have a finite number of positions that are reduced as the volume of samples to be collected increases, which can limit the number of time points that can be collected.

Sample splitters can be used to divert the eluent sequentially between collection and waste, thus reducing the volume of sample to be collected. Nevertheless, this technique cannot totally reduce the volume of sample sufficiently. Sample collection can become disproportionate and nonrepresentative of the sample eluting when trying to collect small samples. Most importantly, samples are analysed after collection, which can be as long as 24 hours later.

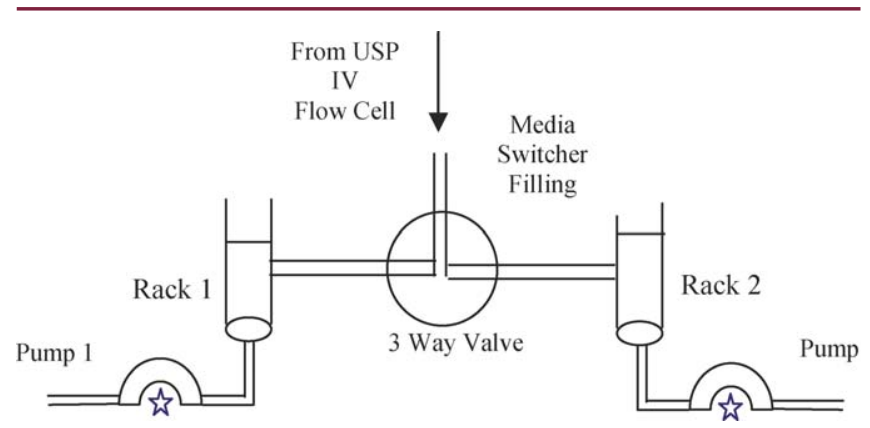

Figure 1: Schematic of the Dual Sampling Rack

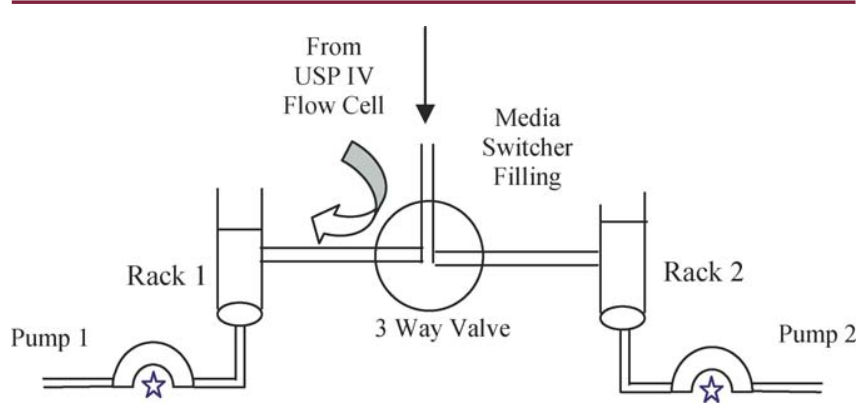

Figure2: Sampling for the first Time Interval

A Dual Sampling Rack ${ }^{\mathrm{TM}}$ has been designed to allow samples to be collected online while at the same time diluting if required and injecting into HPLC or measurement on a UV spectrophotometer.

\section{Dual Sampling Rack Design}

The diagram below shows the design principles of the Dual Sampling Rack ${ }^{\mathrm{TM}}$ for the USP apparatus 4. Each Rack consists of 8 Flow-Through sample compartments. The two racks sandwich a Media Switcher with as many positions as there are sampling compartments.

Each sample compartment is linked to a Pump which can have both forward and reverse directions, and Speed (Figure 1).

At the first Interval, the sample eluent from the USP apparatus 4 is directed into Rack 1 for the duration of the interval (Figure 2).

Immediately the next time interval is due, the " $\mathbf{3}$ Way

Valve" on the Media Switcher is switched to Rack 2 to start collection of the next sampling interval. Pump 1 is turned on in the Forward direction for a Time specified by the operator 
which causes mixing. The IDIS EE software, from Icalis Data Systems, synchronises the complete system and controls the Autosampler which transfers a mixed aliquot of sample for the time interval into HPLC vials or test tubes (Figure 3). At the end of sampling for the time interval, the pump for Rack 1 will be reversed to empty this rack.

The system will be delayed until the next interval is due. Samples for the interval will be collected into Rack 1 while Rack 2 will be mixed and sampled. If there is time remaining until the next collection, the system can be programmed to dilute the samples and inject each sample in turn into an HPLC. The software controlling the system uses a Deferred Data Collection timing sequence which monitors the HPLC analysis time and will not inject the next sample until the HPLC analysis time has elapsed. This timing sequence also gives priority to the Collection and Sampling thus making sure that samples are always collected at the correct times.

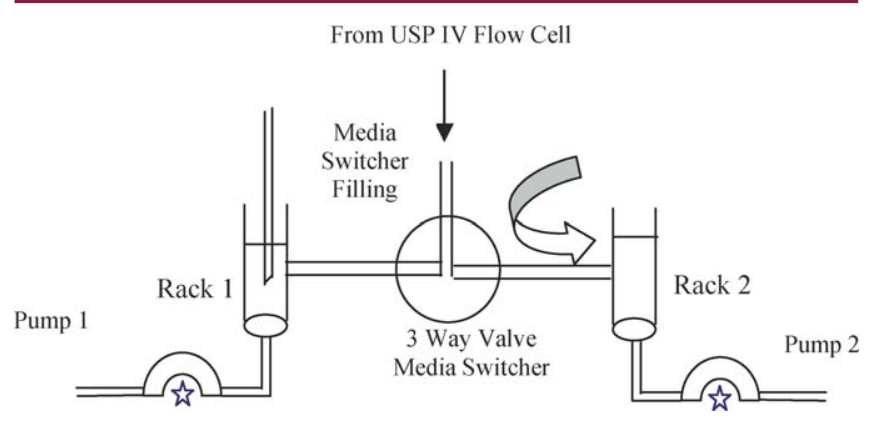

\section{Instrumentation}

The system consists of a USP Apparatus 4 bath linked to a piston pump and to the dual sampling rack on an XYZ Autosampler. A diluter is linked to a single needle probe on the Autosampler and the HPLC column is connected to an injection valve also on autosampler. Sample is pumped continuously throughout the analysis and is switched between the two halves of the dual sampling rack as described earlier. Depending on the dissolution profile and the volume of sample eluent to be collected for each interval, different size racks can be configured. After each injection, an injection contact closure signal initiates the HPLC processing.

\section{Summary}

Using the system described here it is now possible to automate the sequence for collection, sample size reduction, dilution and injection into most generic HPLC systems utilising many time points. This system can also be used to perform measurement online with a spectrophotometer when dilutions are required so that the dissolution profile results are calculated and displayed in real time. Overall, this will produce analytical results in a much shorter time period since processing is performed during the dissolution time period and most importantly there is no manual transfer of samples by the operator. Samples are collected into sealed septa HPLC vials which will minimise evaporation. The system is robust, easy to configure and program thus realising a relatively simple $Q C$ method.

Figure3: Sampling for the next Time Interval

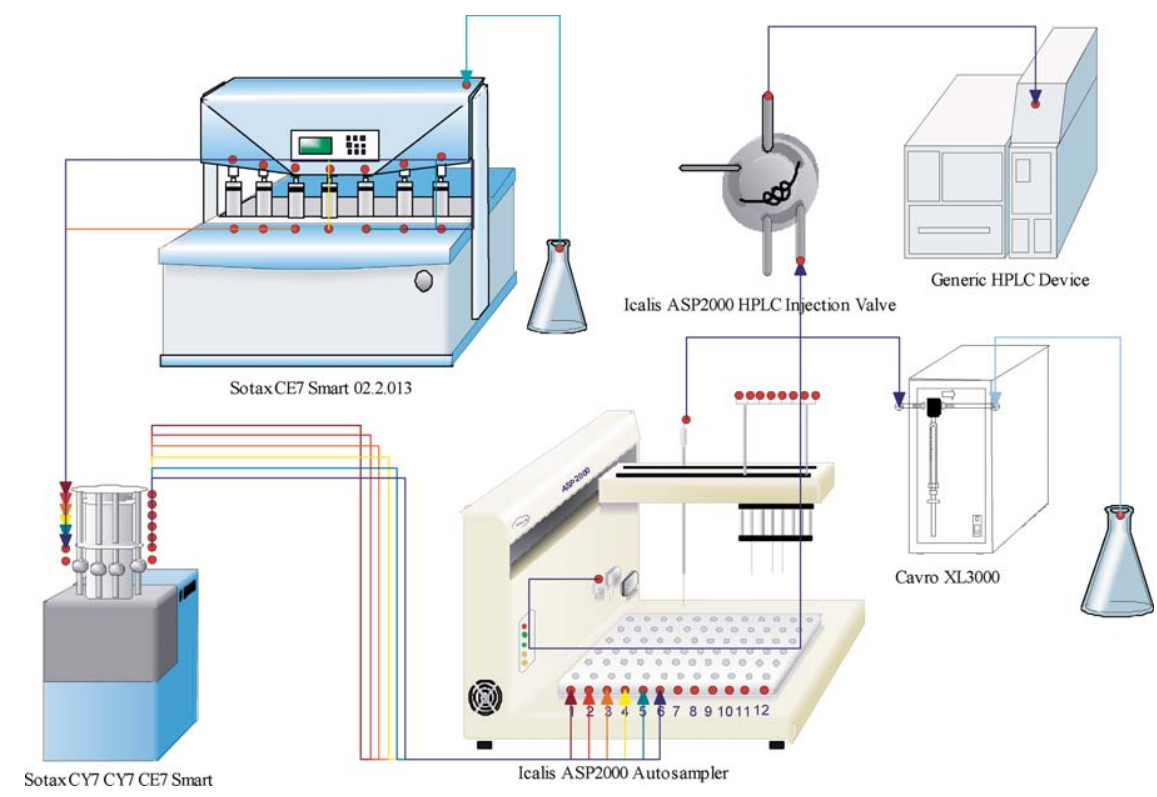

Figure 4. The IDIS EE method configuration using the Dual Sampling Rack with USP Apparatus 4. 